\title{
Urinary tract abnormalities in Chinese rural children who consumed melamine-contaminated dairy products: a population-based screening and follow-up study
}

\author{
Jian-meng Liu PhD, Aiguo Ren PhD, Lei Yang MD, Jinji Gao MD, Lijun Pei PhD, Rongwei Ye MD, \\ Quangang Qu PhD, Xiaoying Zheng PhD
}

Previously published at www.cmaj.ca

$\infty \quad$ See related commentary by Tang, page 424

\section{ABSTRACT}

Background: Kidney damage related to consumption of melamine-contaminated dairy products by young children in China has been described. However, no studies have reported on the population-based prevalence of kidney damage among exposed children or on the condition of affected children after follow-up.

Methods: We conducted an ultrasound-based screening in September 2008 of 7933 children younger than 36 months of age who lived in a rural area in China where the dairy products most highly contaminated with melamine were sold. We monitored children who had evidence of nephrolithiasis or hydronephrosis at screening using renal ultrasonography after one, three and six months. We also collected information from the mothers of affected children about consumption of melamine-contaminated products between June and August 2008.

Results: The overall prevalence of urinary tract abnormalities among screened children was $0.61 \%$ ( $95 \%$ confidence interval [Cl] $0.45 \%-0.80 \%)$. The mean exposure dose of melamine was estimated to be 116 (range 36-220) mg per day. Of the 48 affected children, $43(89.6 \%)$ were asymptomatic, 2 had symptoms and were hospitalized, and 3 had symptoms but treatment had been not sought for them. Of the 46 children for whom six-month follow-up information was available, renal abnormalities persisted in 5 children and resolved in the remaining 41.

Interpretation: Among children who underwent screening, $0.61 \%$ showed ultrasonographic evidence of nephrolithiasis or hydronephrosis. Most of the affected children were asymptomatic. The majority of the affected children recovered from the toxic effects of melamine over time without specific treatment. Renal abnormalities remained in $12 \%$ of the affected children.

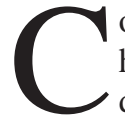
ontamination of dairy products with melamine in China has resulted in a widespread outbreak of serious kidney damage in children. ${ }^{1-4}$ On Sept. 12, 2008, the Chinese government announced to the public that the outbreak had occurred and initiated various emergency responses, including the set-up of a high-level national coordinating group, free screening and treatment of affected children, thorough inspection of all dairy products and producers, timely release of information to the public, recalls of contaminated products, suspension of production of the contaminated milk and compensation to the families of affected children. More than 50000 children have been hospitalized and six have died because of kidney damage. ${ }^{5}$

Melamine is a nitrogen-containing compound commonly used in chemical industry. Because its addition to milk elevates apparent protein content, raw milk was intentionally adulterated with melamine in the production-chain, leading to contamination of dairy products and high-level exposure of thousands of children. ${ }^{6}$

Melamine is known to cause formation of calculi in weanling rats and has led to acute renal failure in cats and dogs consuming melamine-contaminated pet foods. ${ }^{7,8}$ In humans, melaminerelated disease has been recognized only recently, and the full adverse effects of exposure remain unknown. ${ }^{4,9}$ A clinicopathologic study suggests that the size of urinary stones is related to melamine concentration. ${ }^{9}$ A recent hospital-based study in Beijing reported the prevalence of nephrolithiasis was $8.5 \%$ among children who were exposed and referred by other hospitals. ${ }^{10}$

However, no studies have reported on the populationbased prevalence of kidney disease among exposed children, nor have follow-up reports been released about affected children. We performed a population-based screening and followup study involving residents of a rural area situated close to the manufacturer of Sanlu dairy products, which was the source of the most severely melamine-contaminated products in the mainland of China. ${ }^{11}$ The study was approved by the Institutional Review Board of Peking University.

\section{Methods}

\section{Study population}

The area of our study included eight towns in Yuanshi County of Shijiazhuang City, where the Sanlu Dairy Company was located and where its dairy products were distributed. Eligible participants were all children whose mothers

From the Peking University Institute of Reproductive and Child Health (Liu, Ren, Yang, Ye, Qu), Peking University Institute of Population Research (Pei, Zheng), Beijing, China; and the Yuanshi County Centre for Maternal and Child Health (Gao), Shijiazhuang, China

CMAJ 2010. DOI:10.1503/cmaj.091063 
resided in the study area and who were younger than 36 months of age as of September 2008. We obtained a list of children by age and sex from records of birth registration.

\section{Population-based screening}

The administrations of health at different levels of the Chinese government were responsible for providing mass free screening. The Ministry of Health issued guidelines for screening, diagnosis and treatment, and established a national expert group comprising pediatricians, sonographers, nephrologists and radiologists.

In Yuanshi county, a mass screening was conducted during the period of Sept. 15-18, 2008. Through the local health care network, parents of all children under 36 months of age were told to take their children to three designated hospitals for examinations. Experienced and trained sonographers, who were blinded to exposure history, performed ultrasound scans of each child's urinary system. All urinary tract abnormalities were confirmed by a team at the County General Hospital. Mothers of children identified with abnormalities were interviewed about the dairy consumption of their children. Urinary tests for hematuria, leukocyturia and proteinuria were performed.

\section{Follow-up by renal ultrasonography}

We followed up children who had ultrasound findings of nephrolithiasis or hydronephrosis with renal ultrasonography at one month (Oct. 20-23, 2008), three months (Dec. 20-22, 2008) and six months (Apr. 4-5, 2009) after the initial screening. The timing of these follow-up visits was based on the guidelines issued by the Ministry of Health. ${ }^{12}$

\section{Ultrasonographic evidence}

Urinary tract abnormalities included nephrolithiasis and hydronephrosis. We defined nephrolithiasis as increased echogenecity in the renal pelvic. The stones were not uniformly radio-opaque on the ultrasound image. We defined hydronephrosis as an anteroposterior diameter of the renal pelvis of $10 \mathrm{~mm}$ or greater. The ultrasound images obtained at the second and third follow-ups were reviewed independently by a senior sonographer in Beijing, with a rate of concordance of $87.3 \%$. All ultrasound findings reported by county sonographers were confirmed (positive predictive value $100 \%$, negative predictive value $83 \%)$, and a kappa statistic $(\kappa=0.71, p$ $<0.001$ ) showed highly significant reproducibility between the senior sonographers and the local sonographers.

\section{Melamine exposure among affected children}

Dairy products that were contaminated with melamine included 22 brands on a list posted by the Chinese government of 69 batches of contaminated formula. ${ }^{11}$ Children who had consumed a product from any of these 22 brands were regarded as having been exposed to melamine.

Between Sept. 29 and Oct. 1, 2008, we administered a questionnaire and follow-up procedures. At the first followup, we interviewed mothers of affected children about consumption of melamine-containing products between June and August 2008. We asked about product brand, average daily intake and duration of continuous consumption. If a child had stopped consuming dairy products cumulatively for one week or longer, the child was defined as a noncontinuous consumer. To estimate daily intake, we asked two questions, which were "On average, how many cups of milk did your child take per day?" and "How many spoons of powdered formula did you use for one cup of milk?" A 4.3-g spoon had been provided with dairy products. We also asked mothers about their children's symptoms and dates of onset.

\section{Statistical analysis}

For each affected child, we calculated the number of observed person-days from the date of the child's initial abnormal ultrasonography to the follow-up date when ultrasonography was normal, or Apr. 5, 2009, whichever came first. We censored observations of children who had neither nephrolithiasis nor hydronephrosis at follow-up.

We calculated prevalence estimates with exact binomial 95\% confidence intervals $[\mathrm{CI}]$. We calculated overall prevalence using the number of children screened as the denominator. Because information on age and sex had not been collected for children who were screened, we estimated the denominator based on birth registration records.

We used a Kaplan-Meier analysis with log-rank test to compare the cumulative rate for different categorical variables of reversion to normal ultrasonography among children with initial urinary tract abnormalities. We used the proportional-hazard model to look simultaneously at the effects of several variables of interest on the cumulative rate of reversion to normal ultrasonography. These variables included residence (Huaiyang or other towns), maternal occupation (farmer or other) and education (high school and above, or other), and child's sex (male or female), age (younger than 12 months, or 12-36 months old), birthweight (less than or equal

Table 1: Prevalence of urinary tract abnormalities by age and sex among children who consumed melamine-contaminated dairy products

\begin{tabular}{|c|c|c|c|c|c|c|}
\hline Age, mo & \multicolumn{3}{|c|}{ Boys } & \multicolumn{3}{|c|}{ Girls } \\
\hline $12-23$ & 2048 & 11 & $0.54(0.27-0.96)$ & 1735 & 8 & $0.46(0.20-0.91)$ \\
\hline $24-36$ & 1805 & 12 & $0.66(0.34-1.16)$ & 1439 & 8 & $0.56(0.24-1.09)$ \\
\hline
\end{tabular}

Note: $\mathrm{Cl}=$ confidence interval. 
to the median of $3050 \mathrm{~g}$, or higher than $3050 \mathrm{~g}$ ), intake of Sanlu milk products (less than or equal to the median of $86 \mathrm{~g}$ per day, or higher than $86 \mathrm{~g}$ per day), and intake of water (less than or equal to the median of $336 \mathrm{ml}$ per day, or higher than $336 \mathrm{ml}$ per day). We considered a $p$ value of less than 0.05 (two-tailed) to be significant.

\section{Results}

Based on birth records, a total of 9375 children (5114 boys, 4261 girls; age range 0-36 mo) lived in the study area and were thus suspected to have consumed melamine-contaminated dairy products. Of these children, 7933 (4321 boys, 3612 girls) were screened, yielding a rate of screening of $84.6 \%$.

On Apr. 5, 2009, we selected a convenience sample of 24 households across eight towns to investigate why some children were not taken for screening. Of 24 mothers, 18 reported that their children had not or rarely consumed the Sanlu products, four reported that their children were healthy and two reported that they did not have time to attend the screening.

\section{Prevalence at the initial screening}

The overall prevalence of urinary tract abnormalities was $0.61 \%$ (95\% CI $0.45 \%-0.80 \%$ ) among screened children. Among boys, the prevalence was $0.69 \%$, and among girls, it was $0.50 \%(p=0.31)$. Boys were 3.1 times more likely than girls to have a positive finding on ultrasound $(0.56 \% \mathrm{v}$. $0.18 \%, p=0.19$ ) (Table 1), but this result was not significant for children older than one year of age.

\section{Patients at the initial screening}

Forty-eight children (30 boys, 18 girls) had urinary tract abnormalities. Of these children, 24 had nephrolithiasis (diameter 2-9 $\mathrm{mm}$ ) and 24 had hydronephrosis without nephrolithiasis. The demographic characteristics of the affected children are presented in Table 2.

Among the 48 affected children, 43 (89.6\%) were asymptomatic, and five had symptoms, including oliguria and unexplained crying when urinating. The earliest reported date when symptoms were first noticed was May 18, 2008. Two children with symptoms were hospitalized, and the others did not require special treatment. In three patients, results of urinalysis showed abnormalities (hematuria 1, leukocyturia 1, proteinuria 1). No melamine-related deaths were reported in our study population.

\section{Follow-up on affected children}

During up to 2930 person-days of follow-up on the affected children using renal ultrasonography, we observed 41 children whose ultrasound results became normal after they stopped consumption of contaminated milk products. Ultrasonographic evidence of abnormality remained for five children at the six-month follow-up.

The cumulative rate of resolution of abnormalities, as measured by ultrasonography, was $52 \%$ at 48 days after the screening and $88 \%$ at 149 days (Figure 1). We performed univariate and multivariate analyses to assess the associations between resolution of ultrasound abnormalities and characteristics of interest, and did not observe statistically significant associations in any of the models (Table 3).

\section{Exposure to melamine}

Among 69 batches of melamine-contaminated dairy products, 11 were baby formula from Sanlu. ${ }^{11}$ Between January to July 2008 , the median concentration of melamine in these batches was 1351 (range 421-2563) mg/kg. In August 2008, the median concentration had fallen to 1.5 (range $0.7-4.5$ ) $\mathrm{mg} / \mathrm{kg}$.

All 48 affected children had consumed Sanlu products. The median daily intake of the milk powder was $86 \mathrm{~g}$ per day, equivalent to 116 (range 36-220) mg per day of melamine. Two children had consumed other brands in addition to Sanlu; one of these brands was also on the list of products contaminated with

\begin{tabular}{|c|c|}
\hline Characteristic & No. of children $(\%)^{*}$ \\
\hline \multicolumn{2}{|l|}{ Sex } \\
\hline Male & $30(62.5)$ \\
\hline Female & $18(37.5)$ \\
\hline \multicolumn{2}{|l|}{ Age, mo } \\
\hline Mean (SD) & $19.8(8.1)$ \\
\hline $0-11$ & $9(18.8)$ \\
\hline $12-23$ & $19(39.6)$ \\
\hline $24-36$ & $20(41.7)$ \\
\hline \multicolumn{2}{|l|}{ Area } \\
\hline Huaiyang town & $11(22.9)$ \\
\hline Other towns & $37(77.1)$ \\
\hline \multicolumn{2}{|l|}{ Birthweight, $g$} \\
\hline Mean (SD) & $3162(453.0)$ \\
\hline$<2500$ & $1(2.1)$ \\
\hline$\geq 2500$ & $46(95.8)$ \\
\hline Data missing & $1(2.1)$ \\
\hline Birth length, $\mathrm{cm}$, mean (SD) & $50.0(0.7)$ \\
\hline \multicolumn{2}{|l|}{ Gestational age, wk } \\
\hline Mean (SD) & $39.8(1.3)$ \\
\hline$<37$ & $1(2.1)$ \\
\hline$\geq 37$ & $46(95.8)$ \\
\hline Data missing & $1(2.1)$ \\
\hline $\begin{array}{l}\text { Intake of contaminated products, } \\
\mathrm{g} / \mathrm{d} \text {, mean (SD) }\end{array}$ & $89(50.0)$ \\
\hline Intake of water, $\mathrm{mL} / \mathrm{d}$, mean (SD) & $336(196.0)$ \\
\hline \multicolumn{2}{|l|}{ Mother's education } \\
\hline High school or above & $8(16.7)$ \\
\hline Primary school or below & $139(81.3)$ \\
\hline Data missing & $1(2.1)$ \\
\hline Mother's occupation, farmer & $43(89.6)$ \\
\hline
\end{tabular}

Note: SD = standard deviation.

*Unless otherwise indicated. 
melamine. All of the affected children had consumed Sanlu products continuously during the three-month period before they were alerted to the danger of consuming Sanlu products.

\section{Interpretation}

We found that $0.61 \%$ of children had renal stones or hydronephrosis after exposure to a high level of melamine contained in dairy products. During the six-month follow-up period after the initial screening, renal abnormalities disappeared in the majority of affected children without specific medical treatment. However, ultrasound findings remained in $12 \%$ of the children initially diagnosed.

Our study showed that $0.3 \%$ of children exposed to melamine at doses as high as $220 \mathrm{mg}$ per day had renal stones. This rate is much lower than the rate of $8.5 \%$ reported by a tertiary hospital in Beijing. ${ }^{10}$ In the latter, most of the children had been referred for treatment from outside hospitals. A study in Hong Kong identified only one child with renal stones, yielding a prevalence of $0.03 \% .{ }^{13}$ However, the highest level of melamine $(68 \mathrm{mg} / \mathrm{kg})$ in dairy products sold in Hong Kong was only $5 \%$ of the level in Sanlu products. In addition to renal stones, we found that $0.3 \%$ of exposed children had evidence of hydronephrosis. Hydronephrosis can be either congenital or related to renal stones. These factors could not be distinguished in our study. The rate of detection of congenital hydronephrosis in the general population is about $0.17 \% .^{14}$

Most of the children initially found to have either nephrolithiasis or hydronephrosis no longer had evidence of abnormalities six months later. However, our study suggests that renal abnormalities remained in $12 \%$ of the affected chil-

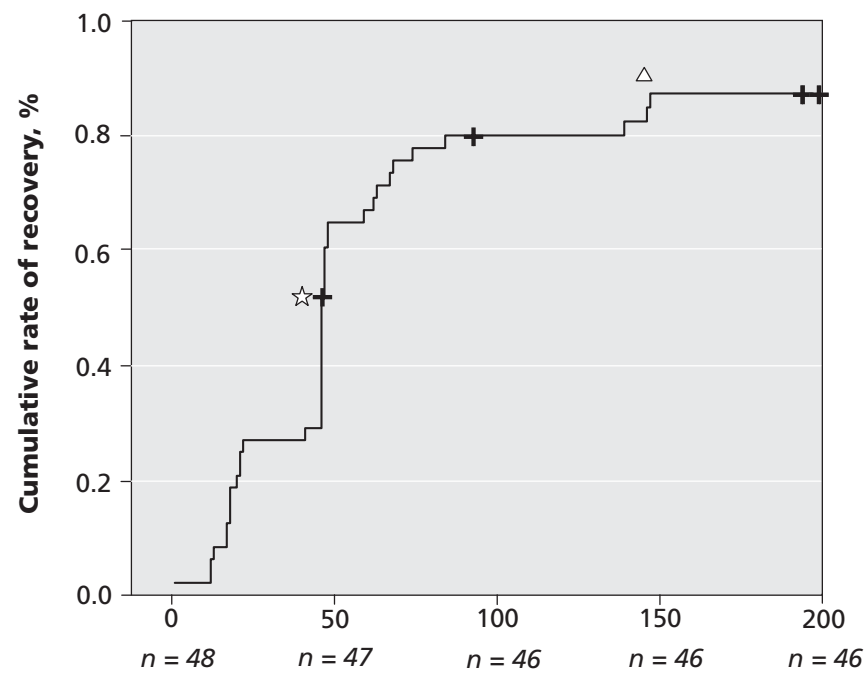

Time since initial screening, days

Figure 1: Cumulative rate of ultrasonographic evidence of urinary tract abnormalities that resolved among children who consumed melamine-contaminated products. Note: $+=$ censored data, star $=52 \%$ of affected children recovered by 48 days since initial screening, triangle $=88 \%$ of affected children recovered by 149 days since initial screening. dren. The potential for long-term complications after exposure to melamine remains a serious concern. ${ }^{4}$ An expert panel sponsored by the World Health Organization, the Food and Agriculture Organization of the United Nations and Health Canada recommended long-term follow-up of children with melamine nephrotoxicity. ${ }^{5}$ Our results suggest a need for further follow-up of affected children to evaluate the possible long-term impact on health, including renal function.

\section{Strengths and limitations}

Among the strengths of our study are its focus on the major distribution area for Sanlu dairy products and its involvement of participants from a naturally living population with the Table 3: Hazard ratios of urinary tract abnormalities that
resolved among children who consumed melamine-
contaminated dairy products

\begin{tabular}{|c|c|c|c|}
\hline Characteristic & $\begin{array}{l}\text { No. of } \\
\text { children }\end{array}$ & $p$ value & $\mathrm{HR}(95 \% \mathrm{Cl})$ \\
\hline \multicolumn{4}{|l|}{ Sex } \\
\hline Male & 29 & 0.085 & $0.52(0.24-1.10)$ \\
\hline Female & 18 & & 1.00 (reference) \\
\hline \multicolumn{4}{|l|}{ Age, mo } \\
\hline$<12$ & 9 & 0.673 & $1.25(0.44-3.57)$ \\
\hline$\geq 12$ & 38 & & 1.00 (reference) \\
\hline \multicolumn{4}{|l|}{ Birthweight, $\mathrm{g}$} \\
\hline$\leq 3050$ & 22 & 0.628 & $0.83(0.40-1.74)$ \\
\hline$>3050$ & 25 & & 1.00 (reference) \\
\hline \multicolumn{4}{|l|}{$\begin{array}{l}\text { Intake of } \\
\text { contaminated } \\
\text { products, g/d }\end{array}$} \\
\hline$\leq 86$ & 27 & 0.418 & 1.00 (ref) \\
\hline$>86$ & 20 & & $1.38(0.63-3.01)$ \\
\hline \multicolumn{4}{|l|}{ Intake of water } \\
\hline$\leq 336$ & 25 & 0.750 & $0.89(0.44-1.82)$ \\
\hline$>336$ & 22 & & 1.00 (reference) \\
\hline \multicolumn{4}{|l|}{ Area } \\
\hline Huaiyang town & 11 & 0.715 & $0.86(0.37-1.98)$ \\
\hline Other towns & 36 & & 1.00 (reference) \\
\hline \multicolumn{4}{|l|}{ Mother's education } \\
\hline $\begin{array}{l}\text { High school or } \\
\text { above }\end{array}$ & 8 & 0.562 & $1.50(0.38-5.91)$ \\
\hline $\begin{array}{l}\text { Primary school or } \\
\text { below }\end{array}$ & 39 & & 1.00 (reference) \\
\hline \multicolumn{4}{|l|}{ Mother's occupation } \\
\hline Farmer & 43 & 0.555 & 1.00 (reference) \\
\hline Other & 4 & & $1.87(0.24-14.82)$ \\
\hline \multicolumn{4}{|l|}{$\begin{array}{l}\text { Abnormalities on } \\
\text { ultrasonography }\end{array}$} \\
\hline Hydronephrosis & 24 & 0.206 & 1.00 (reference) \\
\hline Nephrolithiasis & 23 & & $0.62(0.29-1.31)$ \\
\hline
\end{tabular}

Note: $\mathrm{Cl}=$ confidence interval; $\mathrm{HR}=$ hazard ratio. 
probable highest exposure to melamine worldwide. Therefore, the estimated prevalence of renal damage in our study represents the risk of renal damage in a population after heavy exposure to melamine.

Our study has limitations. First, not all children living in the area were screened, mainly because some parents thought that their children were healthy or had not consumed Sanlu products. To the extent that healthy-appearing children were not brought for screening, the prevalence of abnormalities in our study may have been overestimated. Second, we were unable to collect information on exposure to melamine and demographic characteristics among children with negative ultrasound findings. Third, we relied on maternal recall to assess exposure. Mothers were asked in September to recall milk consumption by their children for the period between June and August. Since the time span for recall was short, our results were not likely to be distorted substantially, if at all, by recall bias. Finally, we did not collect information on breastfeeding practices among affected children. Unpublished data from our institute show that $64 \%$ of infants aged six months are still breastfed, and that baby formula is most commonly given to infants who are not breastfed.

\section{Conclusion}

We found that $0.61 \%$ of children in a rural area of China had renal abnormalities after consuming dairy products that were heavily contaminated with melamine. Without specific medical treatment, the majority of the affected children no longer showed evidence of renal abnormalities within six months after stopping consumption. However, renal abnormalities persisted in $12 \%$ of the affected children, suggesting a need for further follow-up.

\section{This article has been peer reviewed.}

Competing interests: None declared.

Contributors: Jian-Meng Liu, Aiguo Ren, Lijun Pei, Rongwei Ye and Xiaoying Zheng were involved in the conception, design and interpretation of the study. Jian-Meng Liu, Quangang Qu and Lei Yang were responsible for the design of the study. Jian-Meng Liu, Quangang Qu, Lei Yang and Jinji Gao were responsible for the collection of data. Jian-Meng Liu, Lei Yang and Quangang Qu had full access to all of the data in the study and were responsible for the management and analysis of the data. Jian-Meng Liu and Lei
Yang drafted the manuscript. All of the authors revised the manuscript and approved the final version submitted for publication.

Acknowledgements: The authors thank all of the doctors from Yuanshi County Centre for Maternal and Child Health, and two other Yuanshi county hospitals, for their cooperation in this study. They also thank Dr. Xiuzhen Ren, who reviewed the ultrasound images, and Dr. Mary K. Serdula, who reviewed the revised manuscript.

Funding: This work was supported by grants from the National Natural Science Foundation of China and the Major State Basic Research Development Program of China.

\section{REFERENCES}

1. Chan EY, Griffiths SM, Chan CW. Public-health risks of melamine in milk products. Lancet 2008;372:1444-5.

2. Xin H, Stone R. Tainted milk scandal. Chinese probe unmasks high-tech adulteration with melamine. Science 2008;322:1310-1.

3. Parry J. China's tainted milk scandal spreads around world. BMJ 2008;337:a1890.

4. Bhalla V, Grimm PC, Chertow GM, et al. Melamine nephrotoxicity: an emerging epidemic in an era of globalization. Kidney Int 2009;75:774-9.

5. WHO expert meeting to review toxicological aspects of melamine and cyanuric acid. Overall conclusions and recommendations. Ottawa (ON): Dec. 1-4, 2008. Available: www.who.int/foodsafety/fs_management/conclusions_recommendations.pdf (accessed 2009 Dec. 21)

6. Ingelfinger JR. Melamine and the global implications of food contamination. $N$ Engl J Med 2008;359:2745-8.

7. Heck HD, Tyl RW. The induction of bladder stones by terephthalic acid, dimethyl terephthalate, and melamine (2,4,6-triamino-s-triazine) and its relevance to risk assessment. Regul Toxicol Pharmacol 1985;5:294-313.

8. Dobson RL, Motlagh S, Quijano M, et al. Identification and characterization of toxicity of contaminants in pet food leading to an outbreak of renal toxicity in cats and dogs. Toxicol Sci 2008;106:251-62.

9. Lam CW, Lan L, Che X, et al. Diagnosis and spectrum of melamine-related renal disease: plausible mechanism of stone formation in humans. Clin Chim Acto 2009;402:150-5

10. Guan N, Fan Q, Ding J, et al. Melamine-contaminated powdered formula and urolithiasis in young children. N Engl J Med 2009;360:1067-74.

11. Melamine-contaminated baby formula. Beijing (China): General Administration of Quality Supervision, Inspection and Quarantine of the People's Republic of China; 2008 .

12. Guidelines for renal ultrasound examination of children exposed to melamine-contaminated dairy products recommended by the Ministry of Health. Beijing (China): Ministry of Health of the People's Republic of China; 2008.

13. Lam HS, Ng PC, Chu WC, et al. Renal screening in children after exposure to low dose melamine in Hong Kong: cross sectional study. BMJ 2008;337:a2991.

14. Nguyen HT, Kogan BA. Upper urinary tract obstruction: experimental and clinical aspects. Br J Urol 1998;81(Suppl 2):13-21.

Correspondence to: Dr. Jian-meng Liu or Dr. Xiaoying Zheng, Institute of Reproductive and Child Health, Research Center Building, Peking University Health Science Center, 38 College Rd., Haidian district, Beijing 100191, China; liujm@pku.edu.cn orxzheng@pku.edu.cn 